\title{
Mapping Mortality: Using Lexis surfaces to identify complex patterns in age-year specific mortality rates within and between populations
}

\section{Summary}

The paper begins by arguing that much contemporary quantitative analysis of mortality change in populations, such as plotting life expectancy against time, involves 'thinking in slices', analogous to trying to understand complex spatial variation through first plotting (say) mean height as a function of latitude, then as a function of longitude. By contrast Lexis surfaces, a visual method, encourage 'thinking in surfaces', in which the aim of exploratory data analysis is initially not on intelligent data reduction, but on feature recognition.

The paper then illustrates the use of Lexis surfaces through two case studies. In both case studies, three types of Lexis surface visualisation are presented: Shaded Levelplots (SLPS), which facilitate within population comparison; Comparative Levelplots (CLPs), which facilitate comparisons between any two populations; and schematic representations of key features, which facilitate discussion and communication of findings about patterns identified in the first two types of plot.

The paper concludes by discussing some of the substantive implications of features identified in these case studies, and by suggesting that using Lexis surfaces effectively for population data exploration involves iterating between informal and formal, and geometric and aetiological, models of reasoning about population data and the implications thereof. 


\section{Introduction}

This paper will present hundreds of thousands of mortality rates, recorded at over one hundred different ages and over more than sixty years, covering populations in Scotland and those of our nearest neighbours, and selected other populations from across the globe. However it will contain no statistical models, no tables of quantities, and very few numbers. It will do this by presenting a series of maps of demographic data, known as Lexis surfaces, which show how something mortality rates, in this case - varies by both age and by year. Conceptually, Lexis surfaces are to population data as spatial maps are to physical landscapes: in spatial maps, the height of a landscape is shown as a function of both its latitude and its longitude; the Lexis surfaces presented here show mortality as a function of both age and of year. Mortality hazards can be thought to vary continuously over both age and year, much as height varies continuously with both latitude and longitude. And just as it usually makes little sense to try to understand the height of physical landscapes only as a function of their latitude or longitude alone, so this paper will argue that important patterns and features in mortality landscapes are often missed when public health research aims to understand population health, and differences between population, by looking only at how mortality risks vary as a function of age or time alone.

\subsection{Thinking in slices: Life expectancy, age schedules, drift and cohorts}

A life expectancy is a common way that many individual age-specific mortality risks are summarised as a function of year alone. Life expectancies are important summary statistics, and show that, on average, one population tends to live less long than another population in a given year. It is known, for example, that the general trend has been for life expectancies to have increased in richer countries for more than a century. (Leon, 2011) Plotting life expectancies against time for many populations can also show how both relative and absolute inequalities can increase despite outcomes improving for everyone, but at a faster rate for some populations than others. For example, life expectancy at birth, for countries submitting data to the Human Mortality Database (HDM) tended to increase faster for females than males for much of the Twentieth Century, leading to a gap in life expectancy by sex opening up until the 1970s, before starting to narrow again in more recent decades, in large part due to falling rates of cardiovascular disease death in men over 60 years of age. (Glei \& Horiuchi, 2007; White et al., 2014) Similarly, much of Scotland's partly deserved status as 'Sick Man of Europe' is due to its life expectancy trends improving more slowly than those of European neighbours, rather than falling in absolute terms. (McCartney, Walsh, Whyte, \& Collins, 2012; Whyte \& Ajetunmobi, 2012) Throughout the UK, and in England \& Wales in particular, there has been an unprecedented stalling in life expectancies since 2011, leading public health researchers to suggest the effects of austerity are to blame, and for actuarial models to be revised substantially. (Fransham \& Dorling, 2017; Seekings, 2017)

The risk of dying, known as the force of mortality, also varies in characteristic ways with age, according to a 'schedule' that tends to be simple and predictable over some ages, but more complicated and unpredictable at other ages. After around the age of 50, the risk of dying in the next year has tended to increase exponentially with each additional year of age, resulting in a schedule that appears as a straight line when the logarithm of mortality is plotted as a function of age, at least until very old age. Much of this increasing mortality hazard with age has been labelled 'senescent', the results of chronic and cumulative deterioration of bodily structure and function with age, of cells imperfectly copying themselves, each time with some errors, which eventually accumulate and become catastrophic for bodily functioning. Long because the biology of ageing was understood, 
however, the straight line schedule of log mortality against age was noted by insurers, academics, and actuaries, and today is usually referred to as the Gompertz curve, after the author of a publication written in 1825. (Gompertz, 1825) However, not all deaths are caused by old age; others are caused by accidents, violence, childbirth, and other things that 'just happen' to bodies whatever state of repair or disrepair they are in. To represent this hazard of death, a baseline hazard has been added at all ages, producing the next simplest age-mortality schedule, known as the GompertzMakeham model, named additionally after the author of a paper published in an actuarial journal in 1860. (Makeham, 1860) There is also a distinct and high hazard within the first year of life, and within this first year much of the excess mortality is concentrated in the first months, weeks and days. Historically the risk of dying in infancy was similar to the risk of dying at very old age, producing empirical schedules of hazard against age that were high in the first year, low in childhood, then rising again with adulthood, and because of this shape referred to as 'bathtub curves'. Adding yet more complexity to the age schedule is the observation that the 'baseline risk' does not appear constant at all ages, or the same between sexes. Instead, the mortality hazard for the sexes appear to diverge between in the first few years of adulthood: jumping up sharply for males at the start of childhood, largely due to violent and accidental deaths, and historically for females between around the ages of 25 and 30 years, due to the risk of death in childbirth. These additional risks in early adulthood have been described as 'the accident hump', although these deaths are not all accidental. Relatively few models have attempted to parametrically fit the whole age schedule, with the most comprehensive and complicated models, incorporating up to eight parameters being proposed in 1980 (Heligman \& Pollard, 1980), and a somewhat simpler five parameter model, first developed for modelling mortality risks in other animals, being proposed by Siler in 1979 (Siler, 1979), and applied to humans over subsequent years. (Gage \& Dyke, 1986)

Age-specific mortality risks have been constantly changing over time, however, and so any attempts to predict both healthy and overall life expectancies for populations that are not already extinct needs to account for how these age-specific risks will evolve over coming years and decades. Perhaps the most widely used modelling framework for doing this was proposed by Lee \& Carter in 1992, and involves making the assumption that the logarithm of age-specific mortality rates will tend to 'drift' downwards over time at a constant rate. (Lee \& Carter, 1992) Methodologically, it has been noted that the Lee-Carter model is a form of principle component analysis (PCA) which summarises the age-year surface of values using only the first principle component, the 'drift parameter', (Girosi \& King, 2007) and that there may be additional value in characterising populations using additional principle components. (Giordano, Russolillo, \& Haberman, 2008) Conceptually, the Lee-Carter model is based around the Gompertz-Makeham model, assuming its log-linear shape will tend to evolve according to a constant tempo, the 'drift parameter'. For this reason, Lee-Carter models tend to be less effective in estimating mortality rate changes at younger ages (before around age 50), but are often nevertheless relatively effective at estimating future life expectancies because the majority of deaths that occur in richer countries tend to be at these older ages.

An additional complication in thinking about mortality patterns relates to efforts to uniquely model and estimate age-period-cohort (APC) effects. All populations exist and age as cohorts, ageing one year per year, and some specific cohorts appear distinct in their mortality risks than cohorts from neighbouring birth years. For example, people born around the time of the 'Spanish Flu' of 1918 appear to have somewhat higher mortality risks at any given age than might be expected from trends observed in earlier and later cohorts, (Almond, 2006; J Minton, Vanderbloemen, \& Dorling, 
2013) and people born in England and Wales in the 1950s to have a somewhat lower mortality risk as they age than might be expected from broader trends. (Willets, 2003) There have been various attempts to uniquely partition away cohort effects from age effects and period effects in statistical models (for example (Yang, Fu, \& Land, 2004; Yang, Schulhofer-Wohl, Fu, \& Land, 2008)), but doing so is logically impossible, because each of the three effects cannot be uniquely identified (Wilmoth, 2006), leading to effects to do so being branded 'futile'. (Bell \& Jones, 2014)

\subsection{Thinking in surfaces: Visualising and intuiting the geography of mortality using Lexis surfaces}

This paper argues that a more intuitive, and often more productive, way of thinking about and exploring mortality is by visualising log mortality rates by both age and year at once. This can be done by producing level plots, or contour plots, in which year runs across the horizontal axis, age along the vertical axis, and the 'cells' at each age-year 'coordinate' are shaded, coloured, or otherwise marked graphically according to the corresponding age-year specific log mortality hazards. Conceptually Lexis surfaces are maps of age-time much as topographic maps are maps of latitudelongitude. In epidemiology, Lexis surfaces represented as shaded contour maps have been used to identify both mortality surfaces in selected European countries, (J Minton et al., 2013) but their use in demography dates back at least to the 1980s, (Vaupel, Gambill, \& Yashin, 1987) and they are based on the Lexis diagram as discussed in 1875 (Lexis, 1875).

Figure 1a presents a blank Lexis surface, with some additional bands as annotation. The Lexis surface is arranged with equal projection along both axes, such that one year in time is as wide as one year in age as tall. Because of this cohorts run diagonally from bottom left to top right at 45 degree angles. The background of the Lexis surface marks out decades as vertical lines, decadal age groups as horizontal lines, and birth cohort decades as diagonal lines. Within this figure, a specific age band, decade band, and cohort band is highlighted with green, red, and blue colours respectively. In addition to thinking about patterns on Lexis surfaces in terms of bands of parallel horizontal (age), vertical (period) and diagonal (cohort) lines, it is also often useful to look for and identify specific regions within the surface that do not strictly run across the entirety of any of these three planes. These regions can sometimes be usefully interpreted as representing either age-period interaction effects, or age-cohort interaction effects, two examples of which are marked in the figure. Within the demographic time-frame of a century or longer, a common example of an age-period interaction is a war, during which the mortality risks of young adult males tend to rise sharply, before falling back more slowly. Examples of age-cohort interactions are perhaps less common, but potentially include any additional additional mortality risk experienced by 1918 birth cohorts as infants, i.e. the observation of an even higher mortality rate in the first year of life for this cohort even when the whole life-course log mortality multiplier associated with membership of this cohort is taken into account. Particularly high mortality and morbidity risks in the first year of life can be signals of one of the main mechanisms through which persistent life-course cohort effects emerge, namely through interuterine and perinatal exposure to deleterious environments at these earliest life-course stages, often referred to as a Barker Effect, (Barker, 2004) after research by Barker and colleagues demonstrating links between low birthweight and a range of early onset morbidities in adulthood. (Gluckman, Hanson, \& Pinal, 2005; Hales \& Barker, 1992) The importance of later stages in the life course, beyond infancy, for either establishing or accelerating mortality and morbidity hazards at later age, has also been recognised in life-course epidemiology, (Ben-Shlomo, 2002) and ongoing research into the associations between deprivation, gender and cohort membership in Scotland seems to highlight the importance of young adulthood as an important life-course stage, and the macroeconomic conditions established young adults are exposed to at this stage, in establishing 
persistently elevated hazards of suicides and drug-related deaths throughout the later life-course. (J Minton, 2017a; Parkinson, Minton, Lewsey, Bouttell, \& McCartney, 2017a, 2017b) Given the role of critical stages in life-course epidemiology, the value of identifying changes in mortality risk at younger age, as potential determinants of changes in older ages, therefore seems clear.

Figure $1 \mathrm{~b}$ presents an impressionistic schematic of different patterns and trends that are often observed within Lexis surfaces of log mortality rates for rich world populations. Regions within this surface are coloured blue where trends in age-specific mortality tend to be improving, red where they tend to be worsening or relatively bad, and purple where the trends are more inconsistent over time and population. Arrows point upwards to indicate these improving trends, as on Lexis surfaces the corresponding 'bands' of mortality hazards appear to be moving upwards over time, and downwards where the mortality rates are high and/or worsening. For researchers concerned predominantly with life expectancy alone, the two most important age-year regions to note are at the bottom and top of the map, labelled 'Falling Infant Mortality' and 'Lee-Carter Drift'. Mortality hazards within the first age of life have tended to fall, exponentially, since the early twentieth century in most European and North American countries; improvements in many other regions of the world have tended to have started later, but occurred even more quickly, over the middle and later parts of the twentieth century and beyond. Though the force of mortality in the first year is still much higher than over the next few years of age, it is a small fraction of levels that were typical and unchanged for the vast majority of recorded human history.

Because of this fall in infant mortality, the importance of Lee-Carter Drift as a primary determinant of future longevity has tended to rise. On the schematic of Figure $1 \mathrm{~b}$ this is represented as applying mainly from around the age of 50 years, and until old age, but not the oldest ages. Below this age range, mortality trends tend to be more erratic, to differ more between populations and change over time. For male populations in particular, such trends may tend to be more responsive to relatively short term macroeconomic, cultural and social changes that occur within particular countries and regional population groups. For this reason, although changes in mortality rates at these younger adult ages may not contribute greatly to the sum total of mortality that occurs in any given year, they can still be important to explore, both as potential signals of period specific societal phenomena, such as violent conflicts and economic recessions, and also - via cohort effects which emerge from exposures to environmental conditions at later life-stage critical periods - as forerunners of lagged effects which may manifest as elevated mortality hazards in older age ('scarring'). Finally, after the first year of age, mortality hazards within childhood tend to be very low, likely lower than at any previous time in human history, and to be continuing to fall; this makes the increase in mortality hazards for males, in particular, in the first few years of adulthood appear all the more abrupt and stark. 


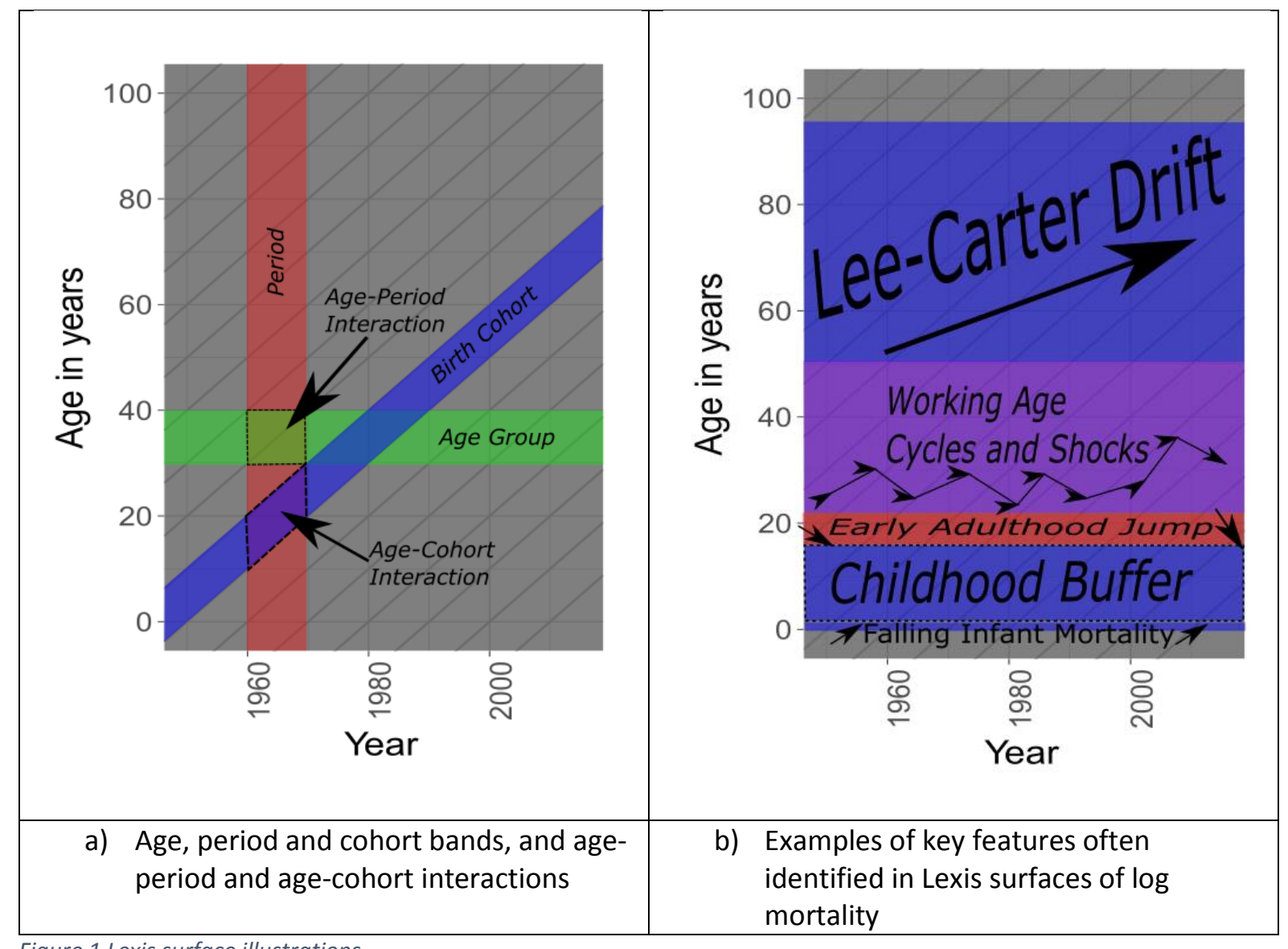

Figure 1 Lexis surface illustrations

\section{Methods}

The data and methods used in this paper are largely as described in a previous paper on quantifying 'excess deaths' in Scotland compared with neighbouring regions over time and at different ages. (Jon Minton et al., 2017) The earlier paper used one year by one year 'Lexis squares' of both exposures (adjusted population counts) and death counts for a range of countries, extracted from the Human Mortality Database (HMD), covering ages in single years up to 109 years old, and years from 1950 to the last available period. Exposure and death counts for the German populations were produced by adding those from East and West Germany. In the earlier paper, populations from individual countries were nested into narrower and broader geographic regions, allowing nested comparison of Scottish age-year specific mortality rates to be compared with both our nearest neighbours (the rest of the United Kingdom), and more distant neighbours (the rest of Western Europe). This earlier paper introduced a variation of the Lexis surface, the comparative level plot (CLP), which is essentially a Lexis surfaces of differences in mortality risks between two Lexis surfaces, each covering the same range of ages and years, but for two different populations, $A$ and $B$. Though it can sometimes be more intuitive to present differences between populations on the Lexis surface as ratios (B/A), (Vanderbloemen, Dorling, \& Minton, 2016) CLPs instead are coloured according to differences in $\log$ mortality $(\log (B)-\log (A))$. Log differences of zero are represented by white cells, cells where $B$ has a higher mortality than $A$ by red shades, and where $A$ has a higher mortality than $A$ by blue shades. The magnitude of the difference between $B$ and $A$ is therefore indicated by the degree of shade, with deeper reds and blues indicating greater differences, and lighter shades indicating smaller differences. An advantage of visualising log mortality differences is that mortality 
differences of $A / B$ and $B / A$ will therefore be of the same shade, with $A / B$ appearing as blue as $B / A$ appears red. (For example, 10 and 0.1 can be represented as $10^{1}$ and $10^{-1}$ respectively, and it is the magnitude of these exponents, 1 and 1 , which determine the cells' shade, whereas the sign of the exponents, positive and negative, which determine the cells' colour.)

This paper differs from the earlier paper, however, in three ways: firstly, the previous paper also calculated life-table estimates of the effect of excess Scottish mortality risks at different ages; this paper will focus only on describing and engaging with the Lexis surfaces themselves, and as by design is more impressionistic rather than actuarial in intent. Secondly, this paper will focus as much on presenting Lexis surfaces of log mortality for individual populations, as much as the CLPs showing differences in log mortality between two populations; for brevity, Lexis surfaces of log mortality will be referred to as Shaded Level Plots (SLPs), and Lexis surfaces of differences between two populations as CLPs. Thirdly, the figures within this paper will present all individual age-year specific log mortality values largely 'as-is', without any smoothing of values to make neighbouring values more similar. Such smoothing of neighbouring values often becomes necessary when contour lines are used, as sudden discontinuities within the surfaces tend to a result in maps that are heavily overplotted with contours, obscuring underlying patterns. However, the SLP Lexis surfaces presented here use colour and shade alone, which removes the overplotting issues that result from using contour lines.

Using colour and shade alone often creates a different interpretative risk, however, as illustrated in the famous Checkershadow Illusion, in which two equally dark grey cells either light if surrounded by darker cells, or dark if surrounded by lighter cells, highlighting the perceptual challenge of correctly decoding specific values from slight variations in cell shade. (Jonathan Minton, 2014) To reduce the risk of incorrectly inferring the value of a cell from its shade and colour, a colour scheme designed mainly to work with categorical data from the RColorBrewer package, called 'Paired', is used in the SCPs. (Neuwirth, 2014) This colour scheme comprises a sequence of six qualitatively dissimilar colours - blue, green, red, orange, purple, and brown - and within each of these six colours the palette cycles between a light and then a dark variant: light blue, dark blue, light green, dark green, and so on. Colours and shades between these 12 colour values are then interpolated to produce hundreds of separate colour/shade combinations for the overall palette. Adjacent groups of colours in this palette are dissimilar enough that it becomes possible to identify relatively narrow bands of values in the Lexis surfaces. Log mortality values are mapped to the palette with base 10 , so -1 means $10^{-1}$ and so $0.1,-2$ means $10^{-2}$ and so 0.01 , and so on. A continuity correction of $10^{-5}$ is added to all values because $\log _{10}$ of 0 is negative infinity, and so cannot be plotted.

For the CLPs the RColorBrewer ' $\mathrm{RdBu}$ ' is used. This palette comprises: dark red, light red, white, light blue, and dark blue. The middle of this palette, corresponding to white, is mapped to 0 , no difference in $\log _{10}$ mortality values between populations, with blues implying that the first of the two populations (' $B$ ') being compared has a lower mortality rate than the second of the two populations $(' A$ '), and red implying that $B$ has a higher mortality rate than $A$. The shade of red or blue indicates the size of the difference $\log _{10} B-\log _{10} A$ for each particular age-year cell.

\section{Results}

The results section will first present an SLP for populations in the British Isles, and then a CLP showing how age-year specific mortality rates in Scotland compare with our neighbours. It will then present SLPs and CLPs for a selection of other population groups. In both series of comparisons schematic representations of the figures will be drawn alongside the SLPs and CLPs themselves. These schematic representations contain labelled regions, coloured according to whether the values 
within broadly indicate improvements (blue) or deteriorations (red) in mortality risks (SLPs) or mortality inequalities (CLPS) in the corresponding SLP and CLP regions. Purple shades are used to indicate other regions in the Lexis surfaces that are useful to mention as, for example, they may indicate anomalies with the population data being visualised.

\subsection{Case Study 1: Lexis Plots of the British Isles}

Figure 2a shows the SLP for British Isles populations, and Figure $2 \mathrm{~b}$ presents CLPs for Scotland as compared with each of these other British Isles populations. Figure 3a presents a schematic representation of some key features identified in Figure $2 \mathrm{a}$, and Figure $3 \mathrm{~b}$ a schematic representation of key features identified in Figure $2 b$.

\subsubsection{SLPs for the British Isles}

As should be expected, the overall impression from the SLPs from Figure $2 a$ is that the Lexis surfaces for these neighbouring populations are broadly similar. Each Lexis surface corresponds broadly to the schematic representation of general mortality patterns presented in Figure $1 b$, with infancy distinct from the rest of childhood, childhood distinct from early adulthood, and mortality from around the age of 50 distinct from earlier ages.

Impressionistically, the mortality surface for England \& Wales appears 'smoother' than the other surfaces, with the surface for Northern Ireland appearing the most 'noisy' or 'speckled', and Scotland's and the Republic of Ireland's mortality surfaces appearing somewhat between Northern Ireland and England \& Wales in its degree of apparent smoothness. It should be reiterated that these plots show the data 'as-is', with no additional smoothing applied, and instead this apparent smoothness or otherwise is a result of the population sizes of these four countries or regions, with England \& Wales having the largest total population, and Northern Ireland the smallest. This difference in apparent smoothness may invite almost philosophical questions about what the mortality surfaces of a hypothetical 'Mega Scotland', 'Mega Northern Ireland', or 'Micro England \& Wales' may look like: i.e. how these surfaces would look if the underlying age-year specific forces of mortality observed in these populations were unchanged, but the total population sizes were either much larger in the first two examples, or much smaller in the last example. Pondering this question also raises interesting thoughts about whether data from whole populations should sometimes be thought about as 'samples' from infinitely sized hypothetical 'hyper-populations', and related to this whether and how standard errors for these age-year specific point estimates should be calculated. Given such issues are not the focus of this paper, they will not be addressed further, but are raised for broader consideration and reflection in later research.

Within Figure 3a, two regions are labelled S1 and S2, where ' $\mathrm{S}$ ' indicates 'senescence'. These two regions divide the region of Lee-Carter Drift indicated in Figure $1 \mathrm{~b}$ into broadly two distinct time periods. Within the schematic of Figure $3 a$ this divide is placed at around 1980, but the main point is to highlight that, for many of the populations shown in Figure 3a, the degree of Lee-Carter Drift appears somewhat discontinuous. There appear to be marked differences in the degree of discontinuity between genders within each of the countries/regions mapped. Typically, the Drift appears more continuous throughout the period mapped for females than for males, where the 'two part' S1/S2 structure of mortality shifts is more apparent. The longer and less interrupted period of continuing older adult mortality risks for females in much of the twentieth century, compared with much more modest improvements for males of the same ages over the same periods, is the key reason why mortality gaps in life expectancy increased between genders in many rich world nations, due in large part to historically higher rates of smoking in males, and poorer cardiovascular mortality; conversely, the more rapid improvements in older ages for males have led to a closing in 
the sex gaps in life expectancy. There also appear to be slight differences in when S1 gave way to S2 between countries/regions within the same gender, with perhaps the S2 improvement phase beginning around three or four years earlier for English \& Welsh populations than for Scottish populations. However this is a more speculative inference than for the sex mortality comparisons.

Towards the start of the period, and in particular at pre- Lee-Carter Drift ages, a region is labelled 'DX', shorthand for 'Demographic Transition'. During much of the first half of the Twentieth century there were very rapid improvements in mortality risks at most ages in richer countries, of a degree not realised before or afterwards, and despite the presence of the two World Wars over these two generations. The region 'DX' appears to indicate the last few years of this period of rapid improvement, and is most apparent at these younger adult ages, perhaps in part due to changes in industrial activity towards less hazardous forms of labour for males, and in a sense to less hazardous forms of labour for females as well, due to improved medical care reducing the risk of death from childbirth.

The region labelled ' $C B$ ', or 'Childhood Buffer', is also marked in Figure 3a, and in Figure 2a produces a chevron-like appearance within childhood ages, with colours/shades indicating higher mortality giving way to colours/shades indicating successively lower mortality risks. For all countries/regions, it is apparent that this buffer is more distinct and localised to before adulthood ages for males than for females, with much more consistent and sharp discontinuities after adulthood for males, and more gradual increases for females reaching adulthood.

A somewhat irregular region ' $T$ ' is labelled, short for 'Troubles', the colloquial name given to the civil conflict in Northern Ireland. This is visible in male young adult all-cause mortality at younger adult ages in Northern Ireland, as a patch of dark red colours which appear after around 1972, compared with lighter pink shades, indicating lower mortality risks, at the same ages from the late 1950s to this turning point. These darker red cells at these ages then give way to slightly lighter pinks, and some green cells, over the subsequent decades. A somewhat subtler version of this same 'disruptive' pattern is also visible for Northern Irish females, and no corresponding sudden shift in mortality risks at these ages are visible for the other populations, including for the Republic of Ireland. This finding is the subject of an upcoming paper, (J Minton, 2017b) which also models this pattern and estimates the age distribution and total number of 'excess deaths' that resulted from this conflict using this allcause mortality data alone.

The three final features to discuss are the red diagonal dashed line, which runs from slightly over the age of twenty years, up through S1 and S2; and the two regions R1, and within this R2 ('Recent'). The diagonal red line indicates the 'scarred cohort' of those born around 1918; this is perhaps most visible for populations in England \& Wales, and least visible within Northern Ireland, but in all cases indicates that cohorts born around the time of the Spanish Flu epidemic faced somewhat higher mortality rates as they aged than would be expected by broader trends. For all ages within this age range, with R1, covering around the 2000s, there some indications of age-specific mortality rates either stalling (England \& Wales, Northern Ireland), or worsening slightly (Scotland), for males in particular. Within the Republic of Ireland, by contrast, mortality rates look to have improved slightly instead. Within R2, covering the most recent years, and younger adults, there are hints that mortality rates may be improving, and that this improvement is possibly seen more for both genders. 


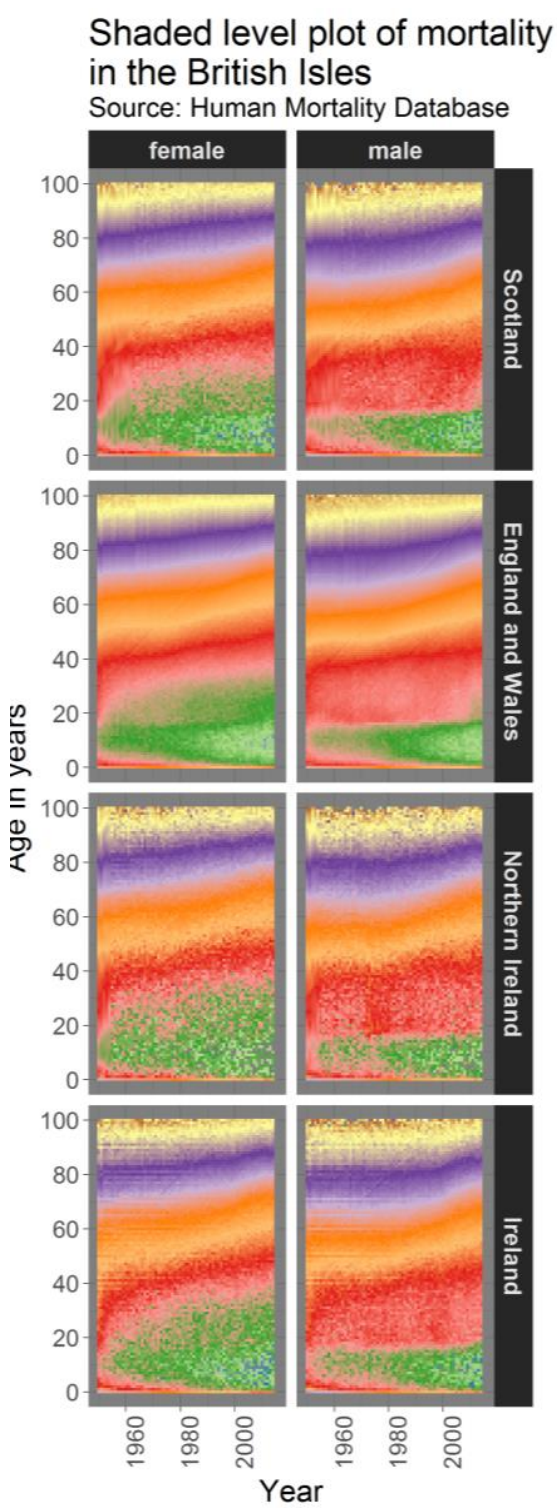

$\log _{10}$ mortality rate $\begin{array}{lllllll} & & & & & & \\ -5 & -4 & -3 & -2 & -1 & 0\end{array}$

a) SCP of Scotland, England \& Wales, Northern Ireland, and the Republic of Ireland

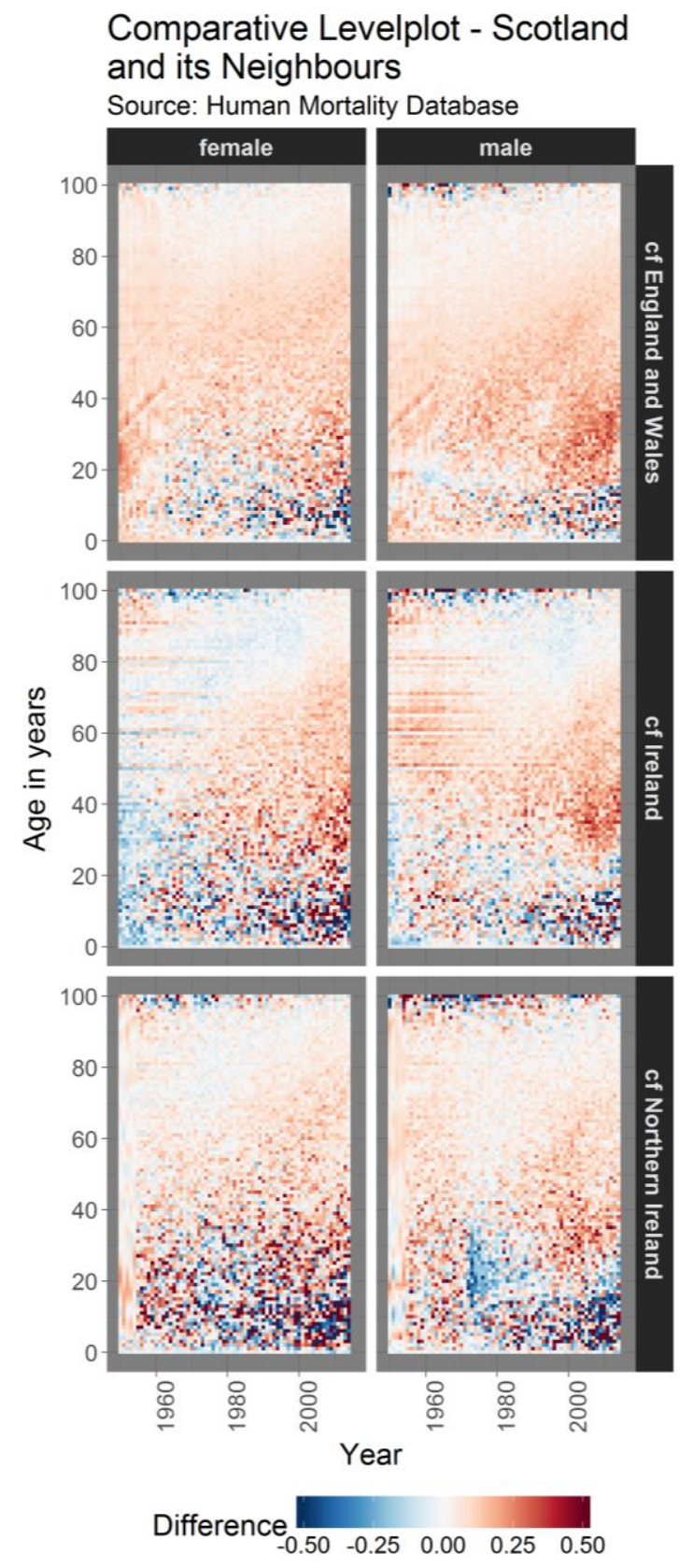

b) CLP of Scotland compared with other populations in the British Isles

Figure 2 Shaded Level Plot of $\log _{10}$ mortality rates for Scotland, England \& Wales, Northern Ireland, and the Republic of Ireland

\subsubsection{CLPs for the British Isles}

The schematic map for discussing the CLP shown in Figure $2 b$ is shown in Figure $3 b$. The purple region marked 'I' (Ireland) corresponds to the horizontal striation visible at many older ages when Scottish populations are compared with corresponding populations in the Republic of Ireland. These horizontal disruptions are also visible in the SLPs for the Republic of Ireland over the same age-year regions as well. Many of these stria appear to be spaced exactly ten years apart, with consecutive bands by single age that are blue-tinged in the CLPs at age 40, 50, 60, 70 and 80 years, then redtinged at age $41,52,61,71$ and 81 years. As these relate to somewhat older data, it appears likely these stria are data artefacts, probably due to either death counts, or population counts, or both, for 
Irish populations up to the 1970 s only being made available to the HMD at five or ten year age groups. The HMD employ a range of methods to interpolate data over these coarser age categories into one year by one year Lexis squares, but such methods will always be imperfect, and the striated appearance of the data appears to indicate this.

The red region 'DX' highlights the observation that, for females aged in their twenties in particular, mortality rates seemed slightly higher in Scotland than in England \& Wales, as well as being lower than corresponding rates in the Republic of Ireland. Above the region marked DX a red diagonal stria is also visible for both genders, up to around the age of 45 years. This could be suggestive that some of the 1918 cohort effect was experienced slightly more severely for Scottish populations than English and Welsh populations; or given this stria disappears after around one decade, it could also be an artefact of, perhaps, slightly less accurate population estimates in Scotland for this cohort than for England \& Wales.

The shape ' $T$ ' ('Troubles') is coloured blue in the schematic, to indicate that mortality rates in young adulthood in Scotland suddenly became relatively better after 1972 than for males of the same age in Northern Ireland. Of course this relative improvement was not due to any improvement in Scottish male mortality at these ages, but the sudden increase in mortality hazards in young Northern Irish adult males. This finding, of sudden apparent comparative improvement in Scottish mortality, was what led to the previously mentioned paper on Northern Ireland being written. (J Minton, 2017b)

The gradated red triangle ' $L$ ', for ' $L a g$ ', indicates a broad tendency firstly for trends in mortality rates improvement to lag slightly behind those of England \& Wales, and secondly for much of this lag to have occurred for generations born after around the 1930s. There are indications of this broad lag operating along generational lines by looking at the changing comparative mortality for both genders in Scotland at around the age of 80 years, which move from lighter to darker red shades, and also noting a fairly subtle triangular pattern in these darker red shades when Scotland is compared with either England \& Wales, or the Republic of Ireland. The transition in comparative mortality trends for Scottish populations compared with Irish is perhaps particularly stark for females, in that mortality risks were historically somewhat lower in Scotland compared with Ireland up to the end of the 1960s, and remained so at older ages up to the late 1990s. This pattern is discussed, and the implications quantified, in more detail elsewhere. (Jon Minton et al., 2017)

The final region indicated in the schematic is ' $\mathrm{R} 1$ ' (Recent), where perhaps the largest comparative relative mortality disadvantages in Scotland are apparent. This appears as a slightly irregularly shaped 'cloud' of darker red shades in both the English/Welsh, and the Irish comparison, and is much more marked for males than females. This recent growth in increased relative mortality disadvantage is also discussed in more detail elsewhere (Jon Minton et al., 2017) and is likely related to worsening trends in some forms of cause-specific mortality in young adult males including suicides and drug-related deaths. (Parkinson et al., 2017a, 2017b) Although it should be noted deaths from these causes and at these ages are relatively rare, a particular source of concern with regard to healthy ageing is if the same factors which lead to elevated mortality risks at these ages also carry over to relative mortality hazard multipliers later in the life course, and so are portents of less healthy aging trajectories for 'Generation X' and later cohorts. 


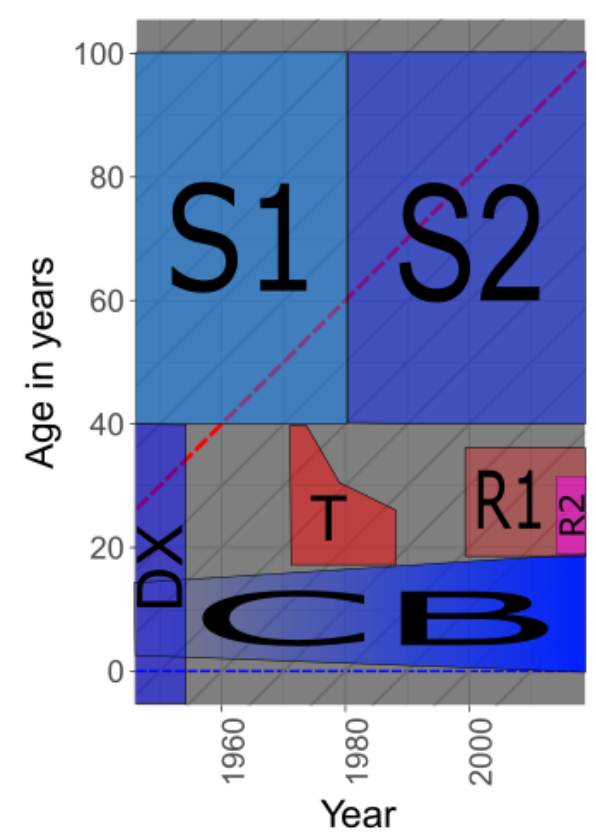

a) Schematic representation of SLPS for British Isles populations

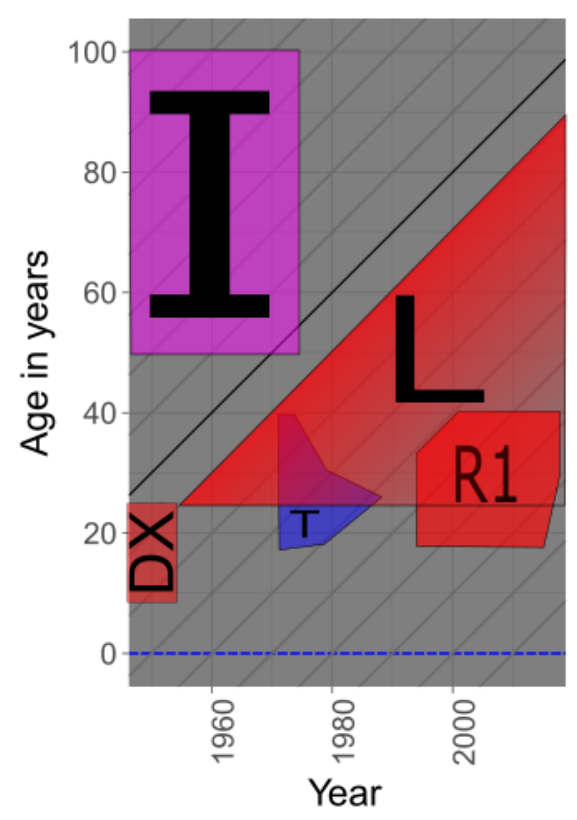

b) Schematic representation of CLPS comparing Scottish populations against other British Isles populations

Figure 3 Schematic representations of SLPS and CLPS for Scotland and other British Isles populations

\subsection{Case Study 2: Lexis Plots of other populations}

This section will produce and discuss features within SLPs and CLPs for some additional geographically defined populations: those of Germany, Taiwan, Japan, Norway, and the United States. These particular SLPs and comparisons are selected mainly to illustrate the broader applicability of the methods beyond understanding mortality trends in the British Isles, and are just a very small fraction of the range of SLPs and comparisons that could be made. The SLPs for these populations are shown in Figure 4a. The populations being compared in the CLPs are i) England \& Wales compared with Germany; ii) Taiwan compared with Japan; and iii) the USA compared with Norway. In each of these examples, cells are blue if the first of the two populations mentioned in the pairings above have lower age-year specific mortality rates than corresponding rates in the second of the two populations, and red if the converse is true. These CLPs are shown in Figure $4 b$, with the SLP for England \& Wales being part of Figure 2a. Schematic representations for each of these SLPS and CLPs are shown in Figure 5.

\subsubsection{SLPs for other populations}

Figure 4a shows the SLPs for Taiwan, Japan, Germany, Norway and the United States. The sub-figure in the top left of Figure 5 presents a schematic representation of some notable features within this figure. Five regions are labelled in this schematic: ' $U 1$ ' and ' $\mathrm{U} 2$ ', ' $C B+^{\prime}$, ' $G$ ', and ' $T$ '. Each of these regions will now be discussed.

The regions $\mathrm{U} 1$ and $\mathrm{U} 2$ (for United States) highlight two distinct red-orange patches visible in young adult male mortality in the USA SLP. The first of these occurs at around the age of 20 years, from around the late 1960s to mid-1970s. The second of these two regions, U2, corresponds to males in their mid to late thirties, in the late 1980s and early 1990s. In both regions, age-specific mortality rates appear slightly higher that might be expected from the broader trends. An arrow is drawn at 
45 degrees from $\mathrm{U} 1$ to $\mathrm{U} 2$ to suggest the possibility that these two regions are linked, as they involve populations drawn from the same birth cohorts. This birth cohorts would have been born in around the decade after World War Two, and on reaching adulthood, would have been exposed both to some of the deadliest stages of the Vietnam War abroad, and to the Counter-culture movement at home. Excess deaths due to both warfare and drugs might therefore be expected in young adulthood, and due to young men but not young women being conscribed to fight, would be expected to affect young adult males more so than young adult females. The region U1 may well be the demographic signal of these events. The later pattern U2 may therefore be linked if earlier life experiences from combat and drug-use had a 'scarring' effects on the mental and physical health of Vietnam veterans, increasing vulnerability to later socioenvironmental hazards. An example of this might be if higher rates of intravenous drug-use by veterans - a cohort effect established at U1 then created additional risk of HIV infection and AIDS related deaths at U2. Exposure to those conditions which gave rise to the apparent excess mortality at U1 could also be linked to increased vulnerability to the heroin, crack cocaine and gang related violence which have come to typify popular narratives of urban life in the USA in the late 1980s.

In contrast to the country and male sex localised pattern of U1/U2, the region marked CB+ indicates an 'extended Childhood Buffer'. This indicates that for Norway, Japan, and Germany, there are indications that low mortality rates, similar to the very low rates of mortality seen in childhood, are increasingly occurring in young adulthood as well, into the mid-thirties, for females but not for males. In these female populations such trends are apparent by noting how the red and pink shades seen in the twenties in the 1950s and 1960s are giving way to greens in the 1980s and 1990s. This 'extended buffer' of low mortality is not observed to the same extent in males, and as a result the sex mortality rate now tends to be higher in young adulthood than at other ages.

The two final features to discuss in the context of the SLPs are G (for Germany), a cohort effect, and $\mathrm{T}$ (for Taiwan), an age-localised period effect. Within the German population SLPs there appear to be at least two nearby cohort-based disruptions, for those aged around 40 years in 1960, and those aged around 43 in 1960. The first of these two parallel lines corresponds to around the 1918 birth cohort, and so to the Spanish Flu effect observed in many other populations. However the latter of the two effects, three or four years later, appears distinct to the German populations, and perhaps results from the particularly severe conditions affecting German newborns in the years immediately following military defeat. The final feature, $\mathrm{T}$, reflects an apparent anomaly in some mortality rate estimates for Taiwanese populations, especially males, aged around 90 years and older, in the late 1970s, which suggest some issues with data quality for these particular years and age groups.

\subsubsection{CLPs for other populations}

This final part of the results section will discuss the three pairs of population comparisons - England \& Wales compared with Germany; Taiwan compared with Japan; and the USA compared with Norway - which are presented on Figure $4 b$, and which are schematically represented within Figure 5. The comparison between England \& Wales, and Germany, will be discussed first, and for the SLPS for England \& Wales see Figure 2a.

\subsubsection{Comparison 1: England \& Wales compared with Germany}

The two regions ED1 and ED2 are, like those for U1 and U2, shown as linked with a diagonal arrow, suggesting the two regions are both linked as different cohorts of a common cohort effect. They are different from the U1/U2 pattern in three ways. Firstly, the ED1/ED2 cohort effect appears to begin at a much earlier stage in the life course, at or near birth. Secondly, the cohort effect appears to be sustained for a longer range of the whole life course. And thirdly, this cohort effect seems to indicate 
lower-than-expected mortality risks for the affected English/Welsh cohorts, whereas the U1/U2 pattern indicates higher-than-expected mortality risks for the affected American cohorts. ED1 indicates that mortality rates in infancy and very young children in England \& Wales were lower than corresponding rates in Germany for those born in the 1950s. ED2 suggests that much of this comparative advantage was then carried to older ages as well, in particular for males. This appears as a broad diagonal blue 'corridor', which stretches to somewhat older ages for males than for females.

This ED2 corridor separates the two regions ED3 and ED4, corresponding in the first case to pre 50s cohorts aged over 50 years, and in the second case to post 1950s cohorts aged between around 30 and 50 years. ED4 is proportionately worse in comparative mortality terms than ED3, though given mortality rates before age 50 are much lower than in older age, are likely to be associated with fewer 'excess deaths' in England \& Wales (compared with Germany) than ED3. However, if the same relative mortality hazards seen in these younger ages in ED4 then continue with these cohorts as they age, the total additional mortality associated with membership of these later cohorts will be much higher.

\subsubsection{Comparison 2: Taiwan compared with Japan}

The second comparison is between Taiwan and Japan, which is shown in the middle row of the CLP in Figure 4b. The schematic representation of this CLP is shown in the bottom left subfigure of Figure 5. Overall, it appears that mortality rates at most ages have tended to be higher in Taiwan than in Japan, with the possible exception of male mortality rates at very old ages. Within this general trend of relative disadvantage, however, are a number of additional features, as shown in the schematic. The first of these features, 'TJ1', focuses again on the apparent data anomaly indicated by region ' $T$ ' in the SLP, i.e. a sudden disruption in log mortality estimates at some of the oldest ages in the mid to late 1970s. Within 'TJ1', it appears as if mortality rates from around age 93 onwards are higher in Taiwan than in Japan from around 1972 to 1975, then for a few years suddenly become lower until around 1980. Again the sudden changes at these age ranges and years suggests there are likely to be particular issues with data quality for these years and age ranges.

A diagonal red line is included in the schematic, separating two broad diagonal polygons labelled 'TJ2' and 'TJ3'. The red diagonal line appears to the cohort aged around 34 years old in 1980, and so born in the wake of the Second World War, around 1945-46. Mortality rates for this cohort, in comparison with Japan, are distinct in that they appear somewhat higher, and this heighted comparative mortality risk appears persistent for both sexes throughout the life course, suggestive of a birth cohort effect. Taiwan was ceded to the Empire of Japan until Japan's defeat in World War Two. With Japan's defeat and US occupation from 1945 onwards, the military strength of Japan's empire fell sharply, and the Republic of China then took control of Taiwan. It appears that Taiwanese populations born into the turmoil near this post War period may have resulted in a Barker Effect on their later mortality risks. Alternatively, there may have been rapid improvements in the environment Japanese infants entered the world after US occupation, leading to faster improvements in Japan than in Taiwan. Though this appears unlikely given the extent of post-War devastation in Japan, only further careful comparison of mortality trends in these and other potential 'control' populations can help untangle the cause of this increased comparative mortality.

The bands 'TJ2' and 'TJ3' therefore correspond to broad cohorts born, respectively, within around one generation before, and within one to two generations after, the end of the Second World War. For both regions, it is clear that mortality rates in Taiwan tend to lag behind those of Japan, but also that the relative additional mortality disadvantage by sex has changed. For the pre-War generation, it appears that the relatively disadvantage in older age is larger for females than males; whereas for 
the post-War generation, the comparative disadvantage appears notably worse for males than for females. It may be that differences by cohort within TJ3 are also important to consider, and in particular that cohorts born in the 1970s appear to have experienced particularly high comparative early years mortality ('TJ5'), then later particularly high comparative mortality in early adulthood ('TJ6'), which may have contributed further to cohort-based disadvantage for these Taiwanese populations. For cohorts born in the 1980 s and beyond, the relative mortality gap in early adulthood seems to have reduced, although it is not yet clear whether relative disadvantage will then emerge again in middle age and beyond.

\subsubsection{Comparison 3: The USA compared with Norway}

The final comparison this paper will consider is between mortality rates in the USA compared with Norway. The CLPs are presented in the bottom row of Figure $4 b$, and the associated schematic in the bottom right subfigure of Figure 5 . It is clear from these figures that mortality rates in the USA tend to have been consistently higher than in Norway for both sexes while of working age ('UN2'). However, mortality rates one or two decades after retirement age tend to compare more favourably between the USA and Norway ('UN1'), with comparative mortality disadvantage giving way to comparative disadvantage between around the age of 70 and 80 years. The age at which the relative mortality disadvantage for US populations disappears may have been increasing, and so for later generations may be lost entirely. The causes of both the relative disadvantage throughout working age, and relative advantage in later retirement age, can only be speculated on; however, it should be noted that, through Medicare, Americans aged 65 years and above have access to healthcare through a single-payer social insurance model not available to working age Americans.

By looking at the very lowest row in the CLP tiles, it is clear that infant mortality rates tend to be higher in the USA than in Norway, and that this relative disadvantage has been increasing over time. Whereas mortality rates in the childhood tended to be similar or lower in the USA up until the early 1980s, after the early 1980s mortality rates also tend to be higher in childhood in the USA than in Norway ('UN3'). 


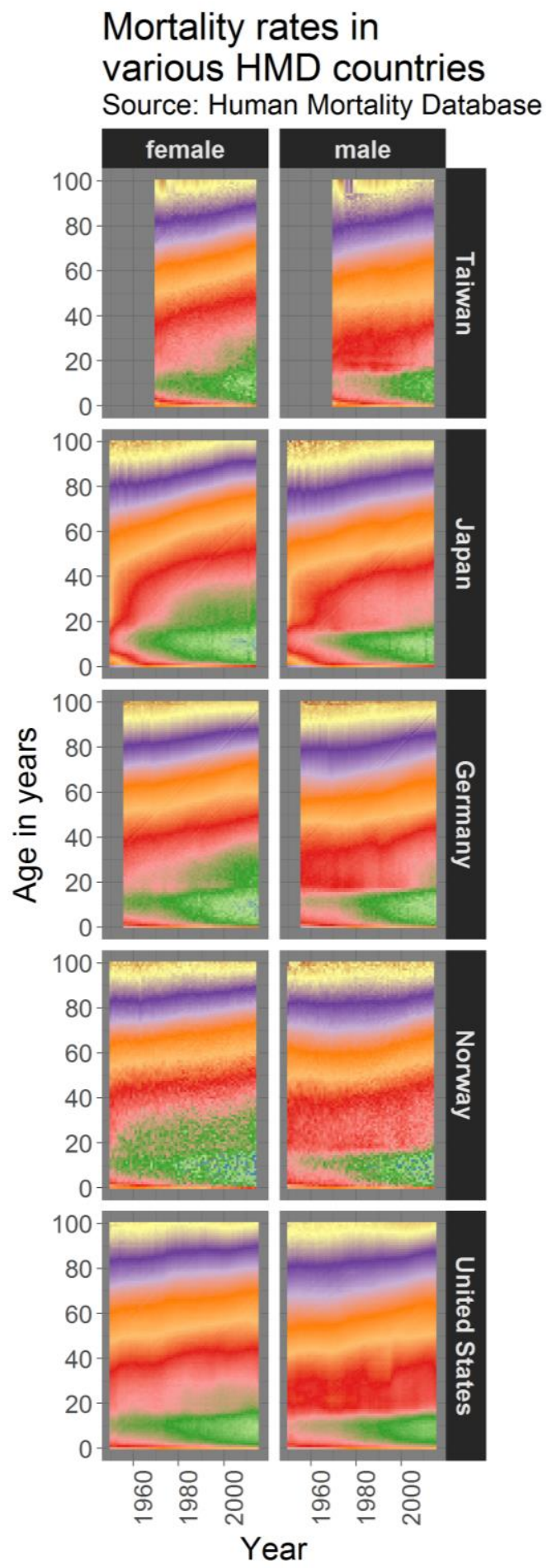

$\log _{10}$ mortality rate $\begin{array}{llllll}15 & -4 & -3 & -2 & -1 & 0\end{array}$

a) SCP of Taiwan, Japan, Germany, Norway, and the United States
Comparative Level Plot various populations

Source: Human Mortality Database
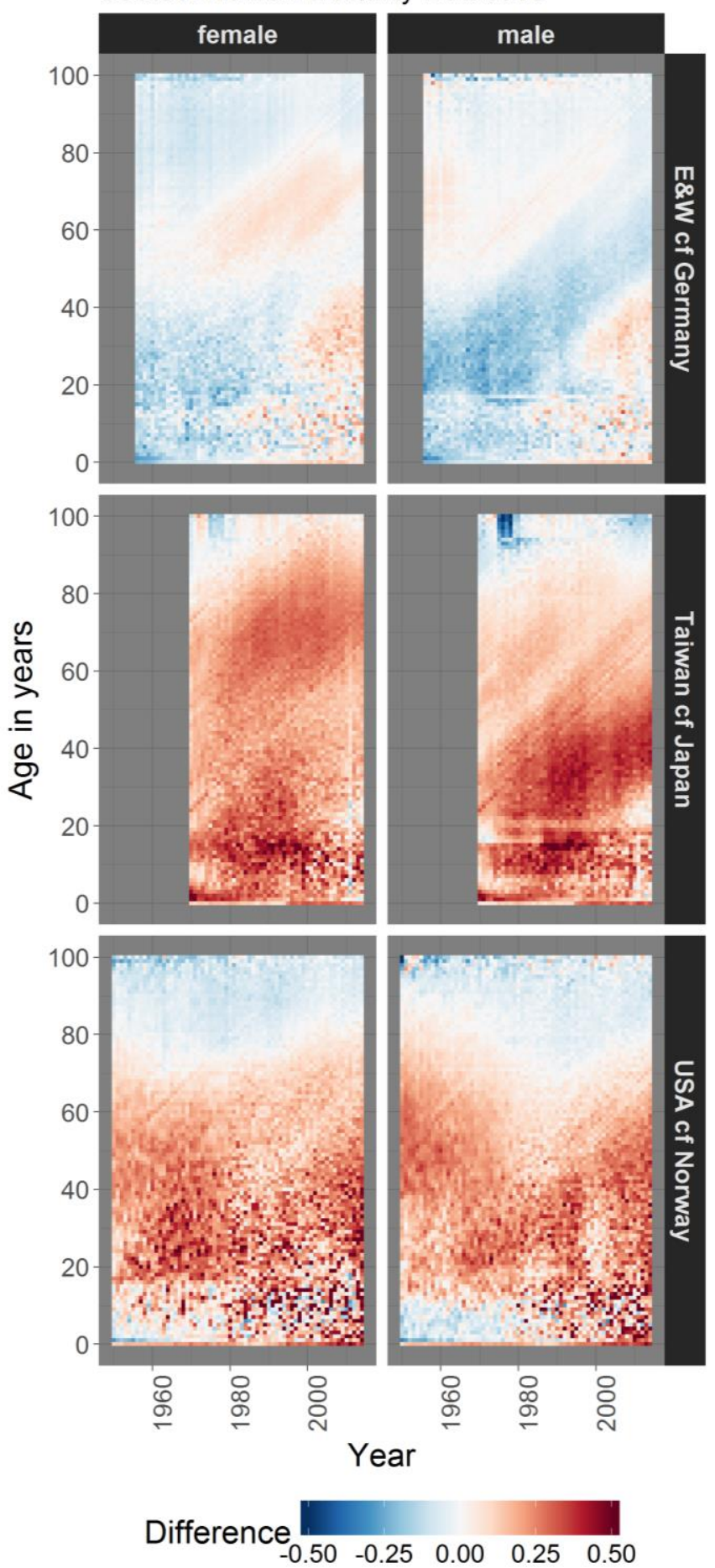

b) CLP comparing log mortality between i)

England \& Wales cf Germany; ii) Taiwan cf Japan; and USA of Norway

Figure 4 a) SCP and b) CLPS for selected additional populations in the HMD 

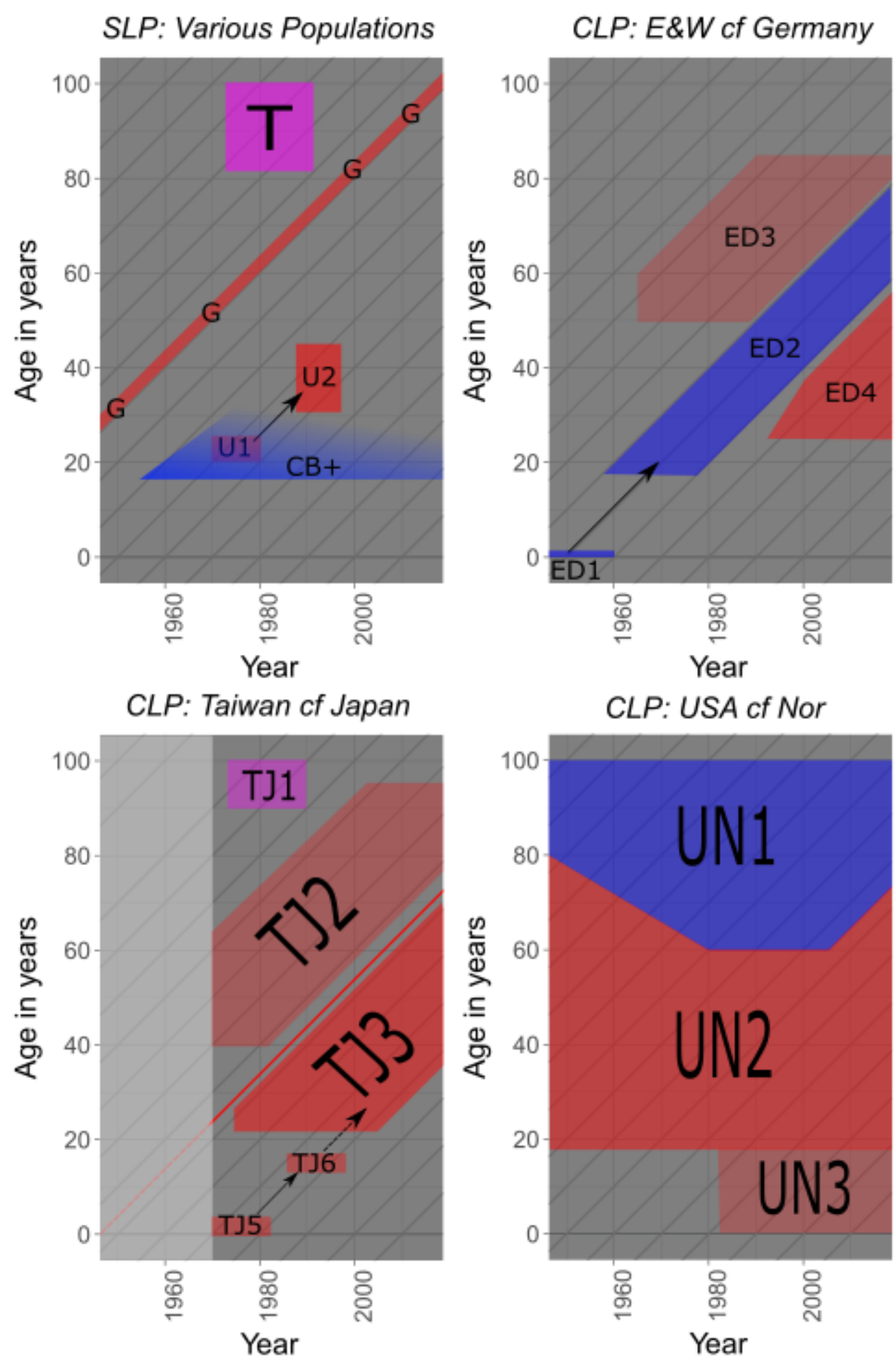

Figure 5 Schematic representations of SLPS (top left) and CLPS for selected populations and comparisons 


\section{Discussion}

This paper has used Lexis surfaces to explore how mortality rates vary with both age and change over time in 18 separate population groups, defined by both gender and geography. Within these populations it has also compared these rates between six pairs of population groups, including three pairs within the British Isles, and three pairs from the rest of the world. Unlike in the previous paper which introduced CLPs, which produced estimates of additional deaths by period and cohort implied by the observed mortality differences, the purpose of this paper has been entirely to illustrate the value of SLPs and CLPs for developing intuitive understandings about how mortality rates compare between populations.

Though this paper has not involved the construction or testing of any statistical models or actuarial tables, it can be argued that the schematic representations of the SLPs and CLPs shown in Figure $1 \mathrm{~b}$, Figure 3, and Figure 5, in which different Lexis surface regions are highlighted and discussed, each represent theoretical postulates about the temporal-geographic features of the Lexis surfaces. It can be useful to draw a broad distinction between the geometric reasoning that Lexis surfaces directly encourage, and the aetiological reasoning that geometric reasoning exists to facilitate; both geometric and aetiological reasoning can be further divided into more informal and formal subtypes, producing the four cell table shown in Table 1. An example of informal geometric reasoning would be to state, "I think I see a 'rectangle' of higher-than-expected mortality in this section of the Lexis surface", whereas an example of formal geometric reasoning would be to construct a statistical model which explicitly represents this geometric feature alongside others identified in the model. An example of informal aetiological reasoning would be to note that the 'rectangle' corresponds to young adult males during a war, and so that the war was responsible for many of the additional deaths, and males of this age were particularly vulnerable to this additional risks because the country had conscription at that time, and young adult men, not boys, were conscripted in this conflict. This aetiological reasoning could then be formalised and operationalised by comparing mortality data for different populations which first entered and exited the conflict in different years, or were exposed to it with different intensities due - for example - to being either nearer or further from the epicentre of the conflict. Models could then be constructed which included different start and end years for different populations, as well as geographical distance parameters to represent these varying population exposures.

\begin{tabular}{|l|l|l|}
\cline { 2 - 3 } \multicolumn{1}{c|}{} & Informal & Formal \\
\hline Geometry & A: Informal Geometries & B: Formal Geometries \\
\hline Aetiology & C: Informal Aetiologies & D: Formal Aetiologies \\
\hline
\end{tabular}

Table 1 Geometrical and Aetiological Reasoning in Lexis surface visualisation

Within two previous papers, more discussion was given to how statistical models may be constructed on the basis of informal geometric reasoning using Lexis surface visualisation, and tested and compared formally in a falsifiable model testing framework. In Table 1, therefore, this earlier paper focused largely on how to strengthen links between the elements in the top row, $A$ and $B$. By contrast, this current paper has been more focused on strengthening links between the elements in the first column, $A$ and $C$. It has done this by using the schematic figures to facilitate discussion of the SLPS and CLPs. The purpose of these discussions has not been to confirm or falsify any particular hypotheses, but simply to point out features within the surfaces. 
The somewhat informal, non-linear and subjective approach to mortality data analysis and population comparison presented here raises a number of broader issues relating to different processes of scientific inference and methodology. A common way of dividing ways of reasoning is into the 'bottom-up logic' of inductive reasoning, and the 'top-down' logic of deductive reasoning. If the approach illustrated here were forced into one of these two methodological camps, it would likely fit more easily as a method of induction than deduction. However, it might be easier to understand the approach from the framework of a third, lesser known, method of reasoning, abduction, developed by the North American logician and Pragmatist C.S. Pierce. (Menand, 2009) Whereas deduction, induction and abduction all possess both explanations (guesses/hypotheses) and explananda (observations/data), it is within abduction that a fuller description of the complex processes by which competing explanations emerge and come to be preferred in response to growing explananda is provided. Put simply, much of abduction seems to be the process of using evidence to choose between schema; in the case of reasoning geometrically about patterns of Lexis surfaces, these schema may take the form of various types of Platonic shapes - triangles, squares, circles and so on - and more complex modifications thereof; the selection of aetiological schema can be considered a linked, parallel process, with different geometric forms suggesting different aetiological schema preferences; and in cases where the geometric shapes are ambiguous, for example semi-diagonal ellipses indicating combinations of age, period and cohort effects, the aetiological schema preferences are also likely to influence selection of geometric schema as well. The schema being considered and preferred in the process of exploring data will also tend to influence which additional data are explored. For example, if the preferred aetiological schema suggest that higher mortality at older ages are the result of a cohort effect due to differential exposure to different labour market or regulatory environments in young adulthood, then the researcher may wish to look next at economic or regulatory statistics for the populations of interest.

Though many of the ideas and guesses that emerge from Lexis surface visualisations are inherently subjective and speculative, they nevertheless seem necessary and sometimes neglected aspects of the broader scientific process. The purpose of this paper has been to illustrate some of the countless potential applications of Lexis surfaces as visual tools in pursuit of effective scientific research into population change, and healthy ageing, in Scotland and beyond. 
Almond, D. (2006). Is the 1918 Influenxa Pandemic Over? Long-Term Effects of In Utero Influenza Exposure in the Post-1940 U.S. Population. Journal of Political Economy2, 114(4), 672-712. Retrieved from http://www.stanford.edu/group/scspi/_media/pdf/Reference Media/Almond_2006_Health and Mental Health.pdf

Barker, D. J. P. (2004). The Developmental Origins of Adult Disease. Journal of the American College of Nutrition, 23(sup6), 588S-595S. http://doi.org/10.1080/07315724.2004.10719428

Bell, A., \& Jones, K. (2014). Another "futile quest"? A simulation study of Yang and Land's hierarchical age-period-cohort model. Demographic Research, 30(1), 333-360. http://doi.org/10.4054/DemRes.2014.30.11

Ben-Shlomo, Y. (2002). A life course approach to chronic disease epidemiology: conceptual models, empirical challenges and interdisciplinary perspectives. International Journal of Epidemiology, 31(2), 285-293. http://doi.org/10.1093/ije/31.2.285

Fransham, M., \& Dorling, D. (2017). Have mortality improvements stalled in England? BMJ, j1946. http://doi.org/10.1136/bmj.j1946

Gage, T., \& Dyke, B. (1986). Parameterizing Abridged Mortality Tables: The Siler Three-Component Hazard Model. Human Biology, 58(2), 275-291. Retrieved from https://www.jstor.org/stable/41463747?seq=1\#page_scan_tab_contents

Giordano, G., Russolillo, M., \& Haberman, S. (2008). Comparing Mortality Trends via Lee-Carter Method in the Framework of Multidimensional Data Analysis. In C. Perna \& M. Sibillo (Eds.), Mathematical and Statistical Methods in Insurance and Finance (pp. 131-138). Milano: Springer Milan. http://doi.org/10.1007/978-88-470-0704-8_17

Girosi, F., \& King, G. (2007). Understanding the Lee-Carter Mortality Forecasting Method. Retrieved from https://gking.harvard.edu/files/abs/lc-abs.shtml

Glei, D. a, \& Horiuchi, S. (2007). The narrowing sex differential in life expectancy in high-income populations: effects of differences in the age pattern of mortality. Population Studies, 61(2), 141-159. http://doi.org/10.1080/00324720701331433

Gluckman, P. D., Hanson, M. A., \& Pinal, C. (2005). The developmental origins of adult disease. Maternal and Child Nutrition, 1(3), 130-141. http://doi.org/10.1111/j.1740-8709.2005.00020.x

Gompertz, B. (1825). On the Nature of the Function Expressive of the Law of Human Mortality, and on a New Mode of Determining the Value of Life Contingencies. Philosophical Transactions of the Royal Society of London, 115, 513-583. http://doi.org/10.1098/rstl.1825.0026

Hales, C. N., \& Barker, D. J. P. (1992). Type 2 (non-insulin-dependent) diabetes mellitus: the thrifty phenotype hypothesis. Diabetologia, 35(7), 595-601. http://doi.org/10.1007/BF00400248

Heligman, L., \& Pollard, J. H. (1980). The age pattern of mortality. Journal of the Institute of Actuaries, 107(1), 49-80. http://doi.org/10.1017/S0020268100040257

Lee, R. D., \& Carter, L. R. (1992). Modeling and Forecasting U.S. Mortality. Journal of the American Statistical Association, 87(419), 659-671. http://doi.org/10.1080/01621459.1992.10475265

Leon, D. A. (2011). Trends in European life expectancy: a salutary view. International Journal of Epidemiology, 40(2), 271-7. http://doi.org/10.1093/ije/dyr061

Lexis, W. (1875). Einleitung in die Theorie der Bevölkerungsstatistik. Strassburg: Karl J Trobner. Retrieved from http://www.worldcat.org/title/einleitung-in-die-theorie-derbevolkerungsstatistik/oclc/27127671 
Makeham, W. M. (1860). On the Law of Mortality and the Construction of Annuity Tables. The Assurance Magazine, and Journal of the Institute of Actuaries, 8(6), 301-310. http://doi.org/10.2307/41134925

McCartney, G., Walsh, D., Whyte, B., \& Collins, C. (2012). Has Scotland always been the "sick man" of Europe? An observational study from 1855 to 2006. European Journal of Public Health, 22(6), 756-60. http://doi.org/10.1093/eurpub/ckr136

Menand, L. (2009). The Metaphysical Club. Harvard Library Bulletin, 20(2), 22-23.

Minton, J. (2014). Real geographies and virtual landscapes: Exploring the influence on place and space on mortality Lexis surfaces using shaded contour maps. Spatial and Spatio-Temporal Epidemiology, 10, 49-66. http://doi.org/10.1016/j.sste.2014.04.003

Minton, J. (2017a). Escaping Flatland in population analysis using Lexis surfaces and as-if-spatial reasoning. A research workflow using data on cause-specific mortality amongst males livign in deprived areas of Scotland. Open Science Federation. http://doi.org/10.17605/OSF.IO/NTZ72

Minton, J. (2017b). The Shape of the Troubles: Visualising and modelling conflict-attributable trends in mortality in young adult males in Northern Ireland. Retrieved from https://osf.io/3pj2f/

Minton, J., Shaw, R., Green, M. A., Vanderbloemen, L., Popham, F., \& McCartney, G. (2017). Visualising and quantifying "excess deaths" in Scotland compared with the rest of the UK and the rest of Western Europe. Journal of Epidemiology and Community Health, 71(5), 461-467. http://doi.org/10.1136/jech-2016-207379

Minton, J., Vanderbloemen, L., \& Dorling, D. (2013). Visualizing Europe's demographic scars with coplots and contour plots. International Journal of Epidemiology, 42(4), 1164-1176. http://doi.org/10.1093/ije/dyt115

Neuwirth, E. (2014). RColorBrewer: ColorBrewer Palettes. Retrieved from https://cran.rproject.org/package=RColorBrewer

Parkinson, J., Minton, J., Lewsey, J., Bouttell, J., \& McCartney, G. (2017a). Drug Related Deaths in Scotland. Retrieved from https://osf.io/ecbpn/

Parkinson, J., Minton, J., Lewsey, J., Bouttell, J., \& McCartney, G. (2017b). Recent cohort effects in suicide in Scotland: a legacy of the 1980s? Journal of Epidemiology and Community Health, 71(2), 194-200. http://doi.org/10.1136/jech-2016-207296

Seekings, C. (2017). An "unprecedented" rise in UK deaths sees improving life expectancy stall. Retrieved November 16, 2017, from http://www.theactuary.com/news/2017/03/anunprecedented-rise-in-uk-deaths-will-see-life-expectancy-fall/

Siler, W. (1979). A Competing-Risk Model for Animal Mortality. Ecology, 60(4), 750-757. http://doi.org/10.2307/1936612

Vanderbloemen, L., Dorling, D., \& Minton, J. (2016). Visualising variation in mortality rates across the life course and by sex, USA and comparator states, 1933-2010. Journal of Epidemiology \& Community Health. http://doi.org/10.1136/jech-2014-205226

Vaupel, J. W., Gambill, B. A., \& Yashin, A. I. (1987). Thousands of Data at a Glance: Shaded Contour Maps of Demographic Surfaces. Laxenburg, Austria. Retrieved from http://user.demogr.mpg.de/jwv/pdf/Vaupel-IIASA-RR-87-016.pdf

White, A., McKee, M., de Sousa, B., de Visser, R., Hogston, R., Madsen, S. A., ... Raine, G. (2014). An examination of the association between premature mortality and life expectancy among men in Europe. European Journal of Public Health, 24(4), 673-9. 
http://doi.org/10.1093/eurpub/ckt076

Whyte, B., \& Ajetunmobi, T. (2012). Still "The sick man of Europe"? Scottish Mortality in A European Context 1950-2010. An analysis of comparative mortality trends. Glasgow. Retrieved from http://www.gcph.co.uk/assets/0000/3606/Scottish_Mortality_in_a_European_Context_2012_ v11_FINAL_bw.pdf

Willets, R. (2003). THE COHORT EFFECT : INSIGHTS AND EXPLANATIONS.

Wilmoth, J. R. (2006). Age-Period-Cohort Models in Demography. In G. Caselli, J. Vallin, \& G. Wunsch (Eds.), Demography: Analysis and Synthesis. Burlington, MA: Academic Press.

Yang, Y., Fu, W. J., \& Land, K. C. (2004). A Methodological Comparison of Age-Period-Cohort Models: The Intrinsic Estimator and Conventional Generalized Linear Models. Sociological Methodology, 34(1), 75-110. http://doi.org/10.1002/cncr.20987

Yang, Y., Schulhofer-Wohl, S., Fu, W. J., \& Land, K. C. (2008). The Intrinsic Estimator for Age-PeriodCohort Analysis: What It Is and How to Use It. American Journal of Sociology, 113(6), 16971736. http://doi.org/10.1086/587154 